\title{
Diseño y simulación preliminar del cubo del rotor para una turbina eólica de 50- kW clase II, de acuerdo a la norma IEC-61400-2
}

\section{Preliminary design and simulation of the rotor hub for a $50-\mathrm{kW}$ class II wind turbine, according to the IEC-61400-2 standard}

COLÍN-MARTINEZ, José Luis †ै*, LÓPEZ-GARZA, Víctor, HERNÁNDEZ-ARRIAGA, Isaac y NAVARRO-ROJERO, María Guadalupe

CIATEQ A.C., Av. del Retablo 150 Col. Constituyentes Fovissste Querétaro, C.P. 76150, Querétaro, México.

ID 1 $1^{\mathrm{er}}$ Autor: José Luis, Colín-Martínez / ORC ID: 0000-0003-3095-4115, CVU CONACYT ID: 879470

ID $1^{\text {er }}$ Coautor: Victor, Lopez-Garza / ORC ID: 0000-0001-9090-9119, Researcher ID Thomson: H-6969-2018, CVU CONACYT ID: 554311, Open ID: 107470673007841597382

ID $2^{\text {do }}$ Coautor: Isaac, Hernández-Arriaga / ORC ID: 0000-0002-0668-8649, CVU CONACYT ID: 45617

ID $3^{\text {er }}$ Coautor: María Guadalupe, Navarro-Rojero / ORC ID: 0000-0001-6326-3025, CVU CONACYT ID: 95394

DOI: $10.35429 / J I D .2020 .10 .4 .1 .13$

Recibido 23 de Marzo, 2020; Aceptado 30 de Junio, 2020

\begin{abstract}
Resumen
Actualmente la energía eólica en México está en crecimiento y lo mismo ocurre a nivel mundial, por lo que se deben de desarrollar proyectos con tecnologías nacionales para la fabricación de componentes de los aerogeneradores, en el presente trabajo se realiza una propuesta del diseño del cubo del rotor para una turbina de 50-kW, el objetivo es hacer una nueva propuesta que mejore el diseño anterior del proyecto P07 del Centro Mexicano de Innovación en Energía Eólica (CEMIEEólico), el cual cuenta con un cubo mecano soldado para un prototipo de turbina de $30 \mathrm{~kW}$. Se realiza además una simulación por medio de análisis del método de elemento finito (FEM) aplicando escenarios de carga con el método simplificado de cargas de la norma internacional IEC 61400-2. En las presentes simulaciones se analizan los casos de carga de la norma que influyen directamente en el cubo, después se simulan en el software de simulación ANSYS para validar el diseño propuesto, analizando principalmente los esfuerzos y las deformaciones. Los resultados obtenidos servirán de referencia para fabricar el cubo y evaluar la viabilidad de llevar a cabo una etapa comercial con miras a realizar componentes nacionales para los parques eólicos.
\end{abstract}

Aerogenerador, Cubo del rotor, Diseño, Simulación

\begin{abstract}
Currently, wind energy in Mexico is growing and the same is happening worldwide, so projects with national technologies for the manufacture of wind turbine components must be developed. In this work, a proposal is made for the design of the hub of the rotor for a $50-\mathrm{kW}$ turbine, the objective is to make a new proposal to improve the previous design of project $\mathrm{P} 07$ of the Centro Mexicano de Innovación en Energía Eólica (CEMIEEólico), which has a welded mechanical hub for a prototype turbine $30 \mathrm{~kW}$. In addition, a simulation is performed through analysis of the finite element method (FEA) by applying certain load elements with the simplified load method of the international standard IEC 61400-2. In these simulations, the load cases of the norm that directly influence the cube are analyzed, then simulated in the ANSYS software to validate the proposed design, mainly analyzing the stresses and deformations. The results obtained will serve as a reference to manufacture the cube and evaluate the feasibility of carrying out a commercial stage with a view to making national components for wind farms.
\end{abstract}

Wind Turbine, Rotor hub, Design, Simulation

Citación: COLÍN-MARTINEZ, José Luis, LÓPEZ-GARZA, Víctor, HERNÁNDEZ-ARRIAGA, Isaac y NAVARROROJERO, María Guadalupe. Diseño y simulación preliminar del cubo del rotor para una turbina eólica de 50-kW clase II, de acuerdo a la norma IEC-61400-2. Revista del Diseño Innovativo. 2020, 4-10: 1-13

*Correspondencia al Autor (Correo Electrónico: jose.colin@ ciateq.mx)

$\dagger$ Investigador contribuyendo como primer autor. 


\section{Introducción}

En los últimos años, el sector de energía eólica en México ha crecido considerablemente con más de 50 parques eólicos en donde se encuentran alrededor de 2400 aerogeneradores operando según la AMDEE (AMDEE, 2018), sin embargo, un área en específico que se desarrolla lentamente es la fabricación de equipos y componentes para la energía eólica. En la actualidad a nivel nacional la tecnología que se desarrolla en el territorio nacional es para aerogeneradores de pequeña capacidad la cual comprende a la generación de $10 \mathrm{~kW}$ a 50 $\mathrm{kW}$ de potencia. Las instituciones que han desarrollado este tipo de proyectos son las siguientes:

$\begin{array}{ll}- & \text { CIATEQ A.C (Aerogenerador de } 350 \mathrm{~W} \\ & \text { y } 30 \mathrm{~kW}) \\ \text { - } & \text { CIATEQ A.C / INEEL (Aerogenerador } \\ & \text { de } 500 \mathrm{~W} \text { ) } \\ - & \text { INEEL (Aerogenerador de } 500 \mathrm{~W} \text { ) } \\ - & \text { UNISTMO (Aerogenerador de } 5 \mathrm{~kW}) \\ - & \text { Instituto Tecnológico de la Laguna } \\ & \text { (Aerogenerador de } 30 \mathrm{~kW})\end{array}$

Por lo anterior, se identifica la necesidad de desarrollo de tecnología eólica mexicana como lo ha sido en años recientes el desarrollo de la Maquina Eólica Mexicana para impulsar el sector eólico y en consecuencia del sector energético nacional, así como diseñar componentes con las características específicas de las regiones de potencial eólico de México, con el fin de aumentar la competitividad a nivel internacional en la fabricación de turbinas de pequeña y mediana potencia.

Actualmente en la unidad de CIATEQ A.C. sede Bernardo Quintana, en el área de turbinas eólicas se trabaja en el proyecto de diseño, fabricación y pruebas de un aerogenerador con una potencia nominal de 30 $\mathrm{kW}$, este proyecto está en la fase de ensamble de sistemas y pruebas finales. Además, se tiene aprobados tres proyectos para la consolidación de esta tecnología, uno de ellos se denomina "Desarrollo de aerogenerador de baja potencia $(<100 \mathrm{~kW})$ con un enfoque integral de introducción al mercado nacional" en el cual se diseñará un aerogenerador comercial de $50 \mathrm{~kW}$.
El aerogenerador que se diseñó de 30 $\mathrm{kW}$, es un prototipo que se encuentra en un nivel de maduración TRL 4. El objetivo actual, es llevar un aerogenerador a una fase comercial con un TRL 7, a fin de diseñar y fabricar sus componentes con una visión de producción, y donde el principal problema a resolver es el desarrollo del rotor diseñado y fabricado bajo esta perspectiva de competitividad en el mercado nacional e internacional. Por lo cual se investigarán las mejores prácticas de diseño y manufactura para realizar una producción en serie a menor costo de producción comparado con el rotor de prototipo.

El diseño previo del sistema del rotor del aerogenerador de $30 \mathrm{~kW}$ que se desarrolló tuvo la finalidad de cubrir la necesidad de un prototipo de turbina eólica. La meta ahora, es realizar un nuevo diseño en donde el enfoque es diseñar los componentes del rotor para fines comerciales, por lo que su costo de producción debe ser competitivo y su manufactura con miras a su producción en serie. Desarrollando estos nuevos componentes, se podrá integrar un paquete de transferencia de tecnología a una empresa del sector energético que le interese explotar el mercado, fabricando los productos a gran escala.

Este trabajo, se enfoca en el diseño preliminar del cubo, el cual es el componente del rotor encargado de transmitir la energía mecánica de las aspas al tren de potencia y a su vez al generador eléctrico, el diseño del componente toma en cuenta distintas metodologías de diseño como o lo son: diseño para la manufactura, material, diseño para el ensamble y el diseño para la calidad. Se planea diseñar el cubo para mejorar el proceso de manufactura que se tiene actualmente en CIATEQ. Además, se pretende comprobar la viabilidad de esta nueva propuesta para bajar los costos de fabricación en manufactura en serie.

\section{Rotor de un aerogenerador}

El rotor es el elemento principal de una maquina eólica, siendo su función principal la transformación de la energía cinética del viento en mecánica utilizable, existe gran variedad de rotores y su clasificación más usual se realiza en función de la disposición del eje. 
El rotor de una turbina puede variar dependiendo de la potencia que se requiere generar eso dependerá del tamaño de las aspas, a continuación, se enlistan los componentes que integran al rotor.

\section{Aspas}

La conversión de la energía cinética del viento al movimiento rotativo mecánico se produce debido a las aspas del rotor, estas piezas tienen una forma aerodinámica la cual cambia el flujo del aire, se normaliza y causa una diferencia de presión. Esta diferencia de presión sobre el aspa crea una fuerza de sustentación que a su vez crea un par en el rotor de la turbina eólica (Warmerdam, 2017-2018).

\section{Cubo}

Es el elemento de una máquina donde se apoya y gira un eje. Es una parte muy importante de los aerogeneradores ya que es aquí donde se apoyan las palas y el rotor y es la única parte externa de los aerogeneradores que gira siendo el transmisor de la fuerza del viento al interior de la góndola (Buenestado, 2017).

\section{Sistema de alabeo}

En los aerogeneradores modernos, la velocidad de giro es regulada a través del sistema pitch. El sistema pitch regula el ángulo de paso de las palas del rotor, generando así un mayor o menor accionamiento de salida en las palas del rotor. El sistema pitch completo, incluido el controlador, está alojado delante en el cubo del aerogenerador (IFM, 2017).

\section{Nariz}

Es simplemente un elemento aerodinámico con forma de punta y que se coloca en el buje. Su misión es la de redireccionar el viento de enfrente del rotor, viento a barlovento, hacia los respiraderos de la góndola y así evitar turbulencias en la parte frontal del rotor (Warmerdam, 2017-2018). Ver Figura 1.

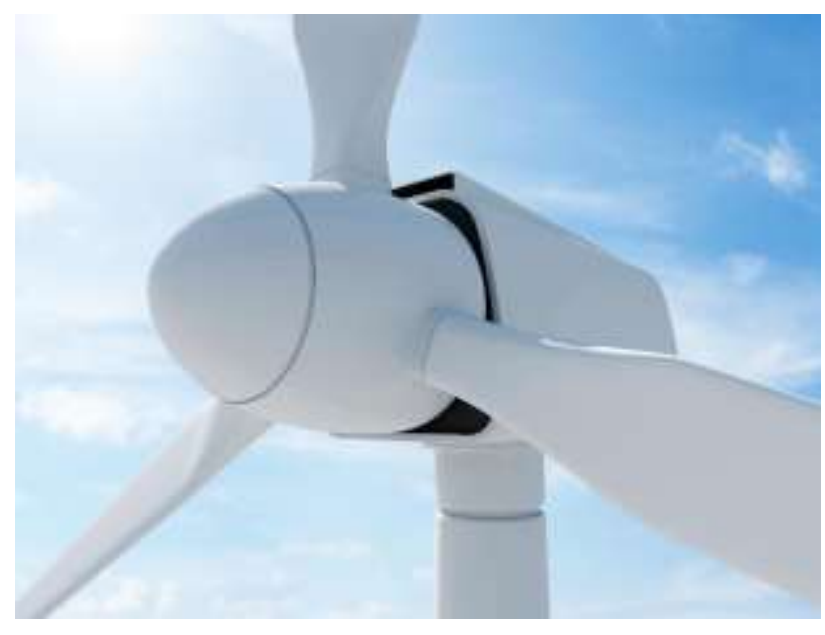

Figura 1 Sistema del rotor ensamblado en una turbina eólica

Fuente: (Scitechdaily, 2020)

\section{Diseño para $X$}

El modelo tradicional de Diseño para X (DFX) es más detallado y amplio que el proceso de diseño tradicional(que considera forma, función, material, proceso) el cual no es tan eficiente como se necesita, la manufactura competitiva requiere el entendimiento preciso de las necesidades del consumidor, las tendencias del mercado, un sistema de diseño de productos competitivos, la utilización de materiales y procesos que lleven a la manufactura de productos de alta calidad, económicos y con rapidez, además de que sean seguros, usables, robustos, fáciles de inspeccionar y mantener. (Anil Mital, 2017)

\section{Diseño para la calidad}

La metodología empleada para esta sección fue el despliegue de la función de la casa de la calidad (QFD), esto para hacer una evaluación de las necesidades del cliente y para conocer el peso de características de calidad del producto para tomar en cuenta en el diseño final de la pieza. A continuación, se muestran las características del perfil del consumidor a seguir, ya que esto ayuda a identificar a los clientes potenciales, es decir, a las personas que puedan adquirir el producto o servicio de CIATEQ:

\footnotetext{
- Empresas que requieran diseños de componentes de turbinas eólicas.

- Empresas e instituciones que quieran realizar manufactura de rotores.

- $\quad$ Empresas que se interesen en adquirir tecnología para su explotación.
} 
- Clientes para crear empresas de base tecnológica.

Como necesidades del cliente se identificaron las siguientes:

$\begin{array}{ll}- & \text { Transferir torque efectivamente } \\ - & \text { Unir componentes del rotor } \\ - & \text { Almacenar componentes de control } \\ - & \text { Sostener el cubo aerodinámico } \\ - & \text { Impulsar el generador eléctrico } \\ - & \text { Conectar sistema de alabeo } \\ - & \text { Proceso de manufactura en serie } \\ - & \text { Bajo coste de fabricación } \\ - & \text { Facilidad de montaje }\end{array}$

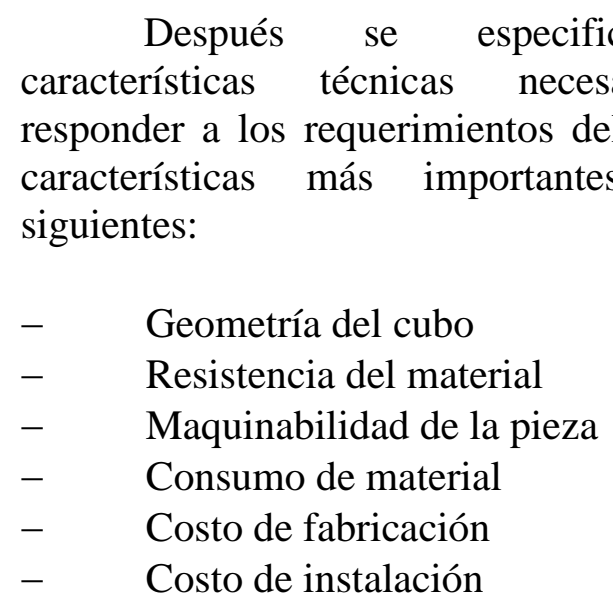

Con estos datos se puede realizar la evaluación de la función de la casa de la calidad, además de la correlación que tiene los requerimientos del cliente y las características técnicas.

\section{Diseño para la manufactura y el ensamble}

Se ha definido la ingeniería concurrente (IC) como un acercamiento sistemático al diseño integrado de productos y los procesos relacionados, incluyendo manufactura y soporte. Pretende que los diseñadores consideren todos los elementos del ciclo de vida del producto, desde el diseño conceptual hasta su disponibilidad en el mercado, incluyendo calidad, costos y los requisitos del usuario.

Una de las metodologías de diseño utilizadas en IC, particularmente las relacionadas con la productividad, es diseño para manufactura (Design for Manufacturing DFM).
Que se puede definir como una colección de programas, técnicas, métrica, herramientas y métodos para mejorar la fabricación de partes o simplificar el ensamble de productos, analizando valores, tolerancias, movimiento, complejidad y conveniencia para el ensamble manual, automático o flexible para que encaje de manera óptima en un sistema de manufactura particular que resulta en costo y calidad excelente. El objetivo de utilizar diseño para manufactura aplicado a un proceso en particular, es diseñar productos que sean fáciles de mantener, confiables, en menor tiempo y que sean más simples, es decir, menos costosos para manufacturar, manteniendo la calidad de los mismos (Claudia Marcela Sánchez R, 2005), los principios de DFM son:

$\begin{array}{ll}- & \text { Reducir el número total de partes } \\ - & \text { Desarrollar un diseño modular } \\ - & \text { Usar materiales y componentes } \\ & \text { estandarizados } \\ - & \text { Diseñar partes multifuncionales } \\ - & \text { Diseñar para fácil fabricación } \\ - & \text { Evitar partes separadas } \\ - & \text { Minimizar las operaciones de } \\ & \text { manipulación } \\ - & \text { Utilizar tolerancias amplias } \\ - & \text { Minimizar el número de operaciones } \\ - & \text { Evitar operaciones secundarias. } \\ - & \text { Rediseñar componentes para eliminar } \\ & \text { pasos de proceso. }\end{array}$

\section{Diseño CAD}

Como primer paso en el proceso de diseño del cubo, se realizó una búsqueda virtual de imágenes, patentes, artículos científicos, etc. estableciendo como fecha de referencia el último año para ver las configuraciones más comunes del cubo. Para el diseño de la geometría del cubo, antes era común el uso de cualquiera de los centros esféricos o estrella, pero hoy en día es posible obtener cubos del rotor topológicamente optimizados. Un algoritmo de optimización se utiliza en FEM para variar el espesor de pared de acuerdo con la tensión local y obtener una solución con el uso mínimo de material. Estas geometrías tienen la forma de una manzana con aberturas adicionales en las zonas donde no se necesita ningún material. La figura 2, muestra la comparación entre el cubo esférico, el cubo de estrella y el cubo topológicamente optimizado. (Krishnappa, 2015) 

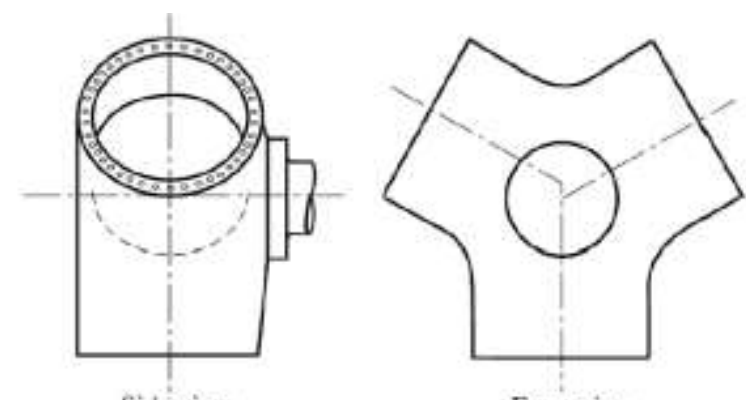

Front view
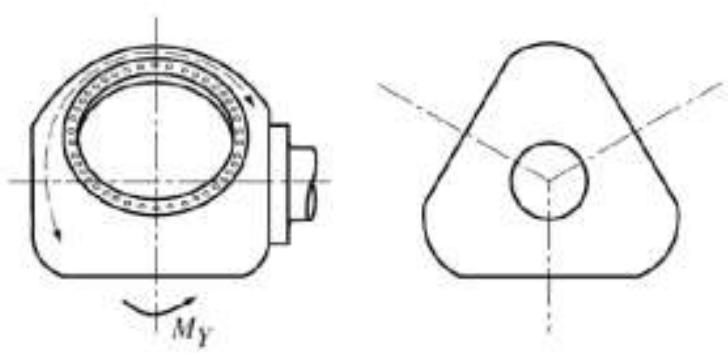

Figura 2 Geometría esférica del cubo Fuente: (Krishnappa, 2015)

Para el diseño CAD del cubo, se utilizó el programa SolidWorks 2016, en el cual la carcasa general del cubo parte de una esfera, el modelo se muestra en la figura 3 , este concepto se utilizó pensando en que la manufactura del cubo se va a realizar mediante fundición de acero, esto disminuirá el costo y el de fabricación comparado con el prototipo anterior.

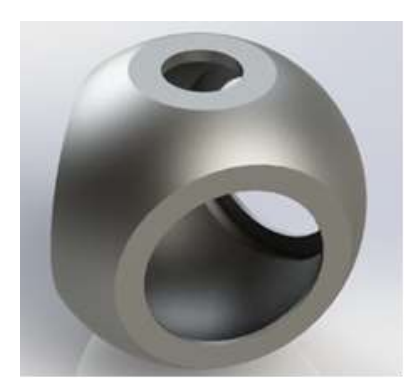

a) Cubo de fundición

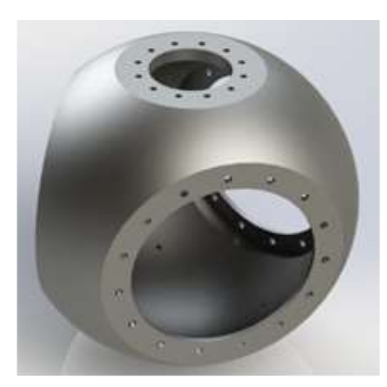

b) Cubo maquinado
Figura 3 Modelo CAD de la propuesta del cubo Fuente: (SolidWorks, 2016)

Para el dimensionamiento de la pieza se tomó en cuenta las proporciones de los sistemas del aerogenerador, para el rotor se utilizará el mismo sistema pitch por lo cual se modelaron los barrenos de las caras en donde ensamblan las aspas, por último, se propuso un diámetro de barrenado para la conexión de la flecha del tren de potencia. La fundición de hierro es el material preferido para construir el cubo. La fundición de hierro se clasifica de acuerdo a sus propiedades mecánicas, tales como resistencia y dureza, en el estándar EN1563.
Los cubos hechos de fundición, son usualmente puestos a prueba mediante ensayos no destructivos con el fin de verificar sus propiedades mecánicas y detectar posibles defectos y discontinuidades internas. Los siguientes son los ensayos no destructivos disponibles y los más usados:

\section{- $\quad$ Inspección por ultrasonido \\ - $\quad$ Inspección por partículas magnéticas \\ - Inspección visual \\ - $\quad$ Medición de dureza.}

Es importante considerar si el material elegido posee la ductilidad necesaria. En ese sentido las bajas temperaturas son críticas para cubos hechos de fundición, en consecuencia, la elección debe hacerse considerando las temperaturas de los alrededores. Tenga en cuenta que las reparaciones del cubo por medio de soldadura no están permitidas (DNV RISO, 2002).

\section{Método simplificado de cargas}

La Norma IEC 61400-2 Parte 2: Pequeños Aerogeneradores, nos propone utilizar el Método de Cargas Simplificadas. Dicho método, hace uso de ecuaciones simplificadas para determinar los momentos y fuerzas generadas en los tres componentes principales del aerogenerador (aspas, torre y eje de baja), de acuerdo a ciertas condiciones que pueda experimentar el aerogenerador durante su tiempo de vida. (IEC, 2013) La Norma propone el diseño de 10 casos de carga, los cuales aparecen en la tabla 1.

\begin{tabular}{|c|c|c|c|}
\hline Situación de diseño & & Hipótesis de carga & $\begin{array}{l}\text { Tipo de } \\
\text { análisis }\end{array}$ \\
\hline \multirow{4}{*}{$\begin{array}{l}\text { Producción } \quad \text { de } \\
\text { energía }\end{array}$} & $\mathbf{A}$ & Operación normal & $\mathrm{F}$ \\
\hline & $\mathbf{B}$ & Orientación & $\mathrm{U}$ \\
\hline & $\mathbf{C}$ & Error de orientación & $\mathrm{U}$ \\
\hline & D & Empuje máximo & $\mathrm{U}$ \\
\hline \multirow{2}{*}{$\begin{array}{l}\text { Producción de } \\
\text { energía de más } \\
\text { ocurrencia de fallo }\end{array}$} & $\mathbf{E}$ & $\begin{array}{l}\text { Velocidad de rotación } \\
\text { máxima }\end{array}$ & $\mathrm{U}$ \\
\hline & $\mathbf{F}$ & $\begin{array}{l}\text { Cortocircuito en la } \\
\text { conexión de carga }\end{array}$ & $\mathrm{U}$ \\
\hline Parada & $\mathbf{G}$ & Desconexión (frenado) & $\mathrm{U}$ \\
\hline $\begin{array}{lll}\text { Carga extrema del } \\
\text { viento }\end{array}$ & $\mathbf{H}$ & \begin{tabular}{|lll}
$\begin{array}{l}\text { Carga } \\
\text { viento }\end{array}$ & extrema del \\
\end{tabular} & $\mathrm{U}$ \\
\hline 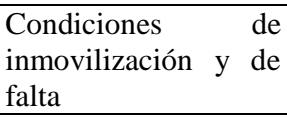 & $\mathbf{I}$ & $\begin{array}{l}\text { Inmovilización con } \\
\text { carga del viento y } \\
\text { exposición máxima }\end{array}$ & $\mathrm{U}$ \\
\hline $\begin{array}{l}\text { Transporte, montaje, } \\
\text { mantenimiento y } \\
\text { reparación }\end{array}$ & $\mathbf{J}$ & $\begin{array}{l}\text { Deberá estar indicado } \\
\text { por el fabricante. }\end{array}$ & $\bar{U}$ \\
\hline
\end{tabular}

Tabla 1 Hipótesis de carga de diseño para el método simplificado.

Fuente: (IEC, 2013) 


\section{Selección de casos de carga}

A primera vista las cargas que actúan en el aerogenerador son bastante simples y obvias. Una corriente de aire en el rotor genera torque y es transferido al generador eléctrico. Una vista más cercana revela más cargas que las supuestas al principio. Junto con las cargas del viento, la estructura completa esta además sujeta a cargas inerciales, gravitacionales y de viento turbulento, el sistema coordenado de la turbina se muestra en la figura 4 , en el cual se aprecia que el eje $\mathrm{Z}$ va en dirección de la torre, los ejes $\mathrm{X}$ y Y forman un plano paralelo al suelo en donde se encuentra la cimentación del aerogenerador. Una de las simplificaciones hechas es pensar que el viento es una corriente constante en todo el rotor. El viento es de hecho altamente no uniforme y su dirección cambiará a través del tiempo. Esto crea fuerzas adicionales y momentos alrededor del cubo las cuales someten al cubo y al chasis a complejos casos de carga.

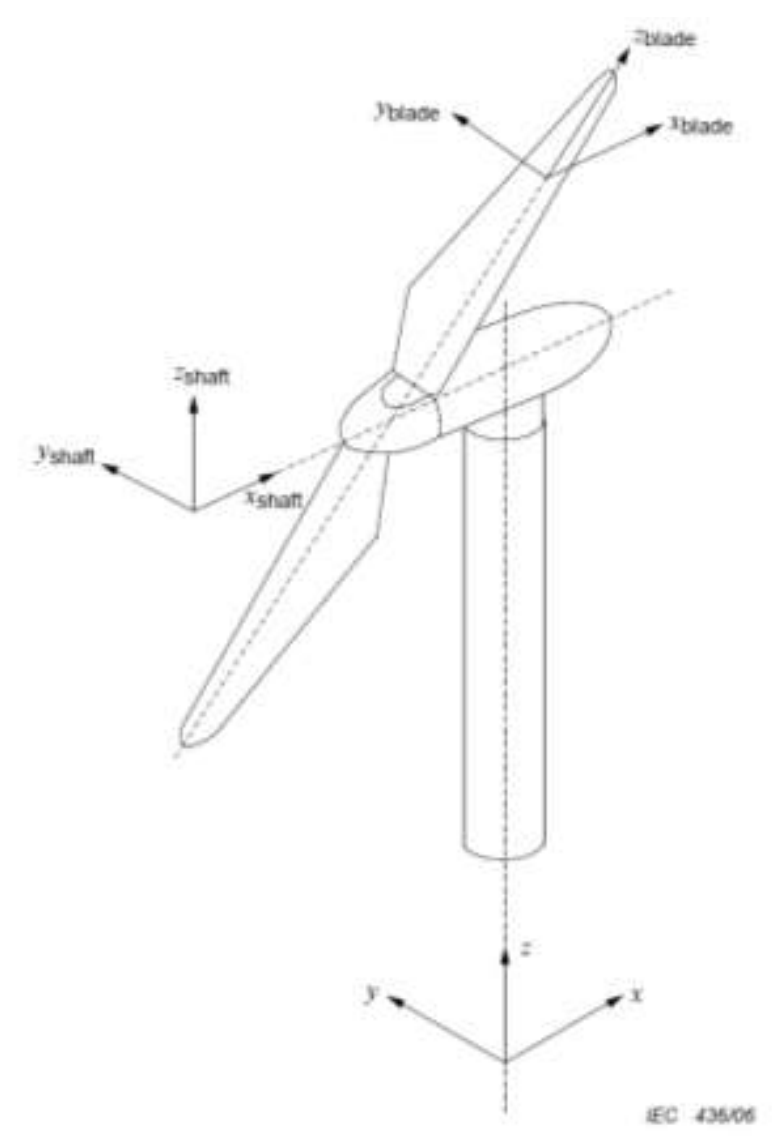

Figura 4 Sistema coordenado de la turbina eólica Fuente: (IEC, 2013)

Dichas cargas provocan esfuerzos en diferentes áreas, y con ello, el máximo esfuerzo necesita ser identificado.
Las aspas experimentarán fuerzas en cada dirección y momentos alrededor de cada eje. Algunas de estas cargas son estables, algunas son cíclicas y algunas son completamente no-cíclicas. Esto hace al aerogenerador una máquina altamente dinámica y sujeta a fatiga.

Los momentos y fuerzas transmitidas al cubo y a la torre dependen de la configuración del cubo. Nos concentraremos en el cubo rígido ya que es la configuración de cubo más utilizada en los aerogeneradores comerciales. El cubo rígido tiene los álabes en voladizo y transmite todos los momentos a la torre (J.F. Manwell, 2009).

Las cargas a considerar para el diseño del cubo deben ser las que actúan en la interacción entre el álabe, eje principal y cubo. Dichos casos de carga son tomados en base a la norma IEC 61400-2 en el apartado correspondiente al diseño de pequeños aerogeneradores. Al analizar la información de los casos de carga del método simplificado, encontramos que los casos críticos en el cubo son los casos A, D y H. Con los resultados de los casos mencionados, se procederá a la simulación y optimización de los conceptos propuestos para el cubo del rotor.

\section{Caso de carga A: Operación normal}

El primer caso de carga cubre el comportamiento a la fatiga durante la operación normal de la turbina. Los rangos considerados en las cargas de fatiga son valores pico a pico (IEC, 2013).

Las cargas están definidas por las siguientes ecuaciones:

Cargas en el Aspa:

$$
\begin{aligned}
& \Delta \mathrm{FzB}=2 \mathrm{mBR} \operatorname{cog} \omega \mathrm{n} 2, \text { design } \\
& \Delta \mathrm{MxB}=\frac{\mathrm{Qdesign}}{B}+2 \mathrm{mBgRcog} \\
& \Delta \mathrm{MyB}=\frac{\lambda \text { designQdesign }}{B}
\end{aligned}
$$

Estos momentos de flexión son para aplicarse en la parte de la raíz del aspa. Las siguientes 3 ecuaciones dan las cargas en el eje de baja velocidad: 
$\Delta \mathrm{Fx}-\mathrm{shaft}=\frac{3}{2} \frac{\lambda \text { designQdesign }}{R}$

$\Delta \mathrm{Mx}-$ shaft $=$ Qdesign $+2 \mathrm{mrger}$

$\Delta \mathrm{Mshaft}=2 \mathrm{mrgLrb}+\frac{R}{6} \Delta \mathrm{Fx}-\mathrm{shaft}$

\section{Caso de carga D: Empuje máximo}

El aerogenerador pequeño puede estar expuesto a cargas de empuje altas en el rotor. Las cargas de empuje actúan en paralelo con el eje del rotor y tiene un valor máximo dado por:

$$
F_{x-s h a f t}=C_{T} 3,125 \rho V_{\text {ave }}^{2} \pi R^{2}
$$
igual a 0.5 .

Donde $\mathrm{C}_{\mathrm{T}}$ es el coeficiente de empuje,

\section{Caso de carga H: Cargas extremas de viento}

Para este caso de carga la turbina está funcionando, durante velocidades de viento extremas como lo es $V e 50$. Para este caso hay dos instancias a considerar, la primera en una turbina parada y la segunda para una turbina en funcionamiento (IEC, 2013).

Cargas de turbina en paro:

$$
\begin{aligned}
& \mathrm{MyB}=\mathrm{Cd} \frac{1}{4} \rho \mathrm{V}^{2} e 50 A_{p r o j, B} R \\
& \mathrm{Fx}-\text { shaft }=\mathrm{B} \times \mathrm{Cd} \frac{1}{2} \rho \mathrm{V}^{2} e 50 A_{\text {proj,B }}
\end{aligned}
$$

Para la turbina en movimiento:

$$
\begin{aligned}
& M_{y b}=C_{l \max } \frac{1}{6} \rho \mathrm{V}^{2} e 50 A_{\text {proje }, B} R \\
& \mathrm{Fx}-\text { shaft }=0.17 \mathrm{~B} A_{\text {proj }, B} \lambda_{e 50}^{2} V_{e 50}^{2} \\
& \lambda \mathrm{e} 50=\frac{\eta_{\max } \pi R}{30 V_{e 50}}
\end{aligned}
$$

Datos de entrada y resultados del análisis simplificado de casos de carga

El método solo aplicará para turbinas de eje

\begin{tabular}{|c|c|c|c|}
\hline Parámetro & Valor & Símbolo & Unidad \\
\hline Densidad del aire & 1.18 & $\rho$ & $\mathrm{kg} / \mathrm{m}^{3}$ \\
\hline Gravedad & 9.81 & $\mathrm{~g}$ & $\mathrm{~m} / \mathrm{s}^{2}$ \\
\hline $\begin{array}{l}\text { Velocidad } \\
\text { promedio de viento }\end{array}$ & 8.5 & Vave & $\mathrm{m} / \mathrm{s}$ \\
\hline Numero de aspas & 3 & $\mathrm{~B}$ & N/A \\
\hline Radio del rotor & 6 & $R$ & $\mathrm{~m}$ \\
\hline $\begin{array}{l}\text { Área proyectada de } \\
\text { las aspas }\end{array}$ & 113.09 & $A_{\text {projB }}$ & $\mathrm{m}^{2}$ \\
\hline $\begin{array}{ll}\text { Coeficiente } & \text { de } \\
\text { arrastre } & \end{array}$ & 1.5 & $C_{d}$ & N/A \\
\hline $\begin{array}{l}\text { Coeficiente de } \\
\text { empuje }\end{array}$ & 0.5 & $C_{T}$ & N/A \\
\hline $\begin{array}{l}\text { Masa del rotor } \\
\text { (HUB + Blade) }\end{array}$ & 860 & $m_{r}$ & $\mathrm{~kg}$ \\
\hline $\begin{array}{l}\text { Velocidad extrema } \\
\text { registrada una vez } \\
\text { en } 50 \text { años }\end{array}$ & 16.16 & Ve50 & $\mathrm{m} / \mathrm{s}$ \\
\hline $\begin{array}{l}\text { Relación de } \\
\text { velocidad en punta } \\
\text { de diseño }\end{array}$ & 5.91 & $\lambda$ design & N/A \\
\hline $\begin{array}{l}\text { Torque del eje de } \\
\text { diseño }\end{array}$ & 70.76 & Qdesign & $\mathrm{Nm}$ \\
\hline $\begin{array}{l}\text { Distancia del } \\
\text { centro de gravedad } \\
\text { del rotor al eje de } \\
\text { rotación } \\
\text { (excentricidad) }\end{array}$ & 0.03 & $e r$ & $\mathrm{~m}$ \\
\hline
\end{tabular}
horizontal teniendo 2 o más aspas en voladizo, con sistemas coordinado de pitch y cubo rígido. Como primer paso se definen los siguientes parámetros de entrada que se muestran en la tabla 2 .
Tabla 2 Parámetros definidos para la realización de los cálculos de casos de carga del método simplificado Fuente: CIATEQ A.C

\begin{tabular}{|c|c|c|c|c|}
\hline \multicolumn{5}{|c|}{ Caso de carga A: Operación normal } \\
\hline Ec. & Descripción & $\begin{array}{l}\text { Valor } \\
\text { SLM }\end{array}$ & Unidades & Símbolo \\
\hline 4 & \begin{tabular}{|l|}
$\begin{array}{l}\text { Empuje en el } \\
\text { eje } \mathrm{x}\end{array}$ \\
\end{tabular} & 104.61 & $\mathrm{~N}$ & \begin{tabular}{|l}
$\Delta \mathrm{F}_{-\mathrm{X}}-$ \\
shaft
\end{tabular} \\
\hline 5 & $\begin{array}{l}\text { Momento en } \\
\text { el eje } x\end{array}$ & 576.95 & $\mathrm{Nm}$ & $\begin{array}{l}\Delta \mathrm{M}_{-\mathrm{X}-} \\
\text { shaft }\end{array}$ \\
\hline \multicolumn{5}{|c|}{ Caso de carga D: Empuje máximo } \\
\hline Ec. & Descripción & $\begin{array}{l}\text { Valor } \\
\text { SLM }\end{array}$ & Unidades & Símbolo \\
\hline 7 & \begin{tabular}{|l|} 
Máximo \\
empuje en el \\
eje $x$
\end{tabular} & 15065.80 & $\mathrm{~N}$ & F_x-shaft \\
\hline \multicolumn{5}{|c|}{ Caso de carga H: Cargas extremas de viento } \\
\hline Ec. & Descripción & $\begin{array}{l}\text { Valor } \\
\text { SLM }\end{array}$ & Unidades & Símbolo \\
\hline 9 & \begin{tabular}{|l|} 
Máximo \\
empuje en el \\
eje x
\end{tabular} & 83337.17 & $\mathrm{~N}$ & F_x-shaft \\
\hline
\end{tabular}

Con los datos anteriores se calculan los momentos y fuerzas con las ecuaciones establecidas anteriormente de acuerdo a la Norma IEC 61400-2, los resultados obtenidos se muestran en la tabla 3.

Tabla 3 Resultados de los cálculos del método simplificado de cargas

Fuente: Elaboración Propia 


\section{Metodología a desarrollar}

Para la selección del material se seleccionó el material de la base de datos del ANSYS, una vez que se obtengan los resultados de simulación se seleccionara el material con las características mecánicas que se requieran para cumplir con los factores de seguridad y el proceso de fabricación de la pieza.

En la simulación se utilizó el software de elemento finito ANSYS Workbench para evaluar cada uno de los casos de carga (A, D y $\mathrm{H})$ el material que se seleccionó fue el acero estructural de la biblioteca del software las propiedades de este material se enlistan a continuación en la tabla 4 .

\begin{tabular}{|l|r|l|}
\hline \multicolumn{1}{|c}{ Propiedad } & \multicolumn{1}{c|}{ Valor } & \multicolumn{1}{c|}{ Unidad } \\
\hline Densidad & 7850 & $\mathrm{Kg} / \mathrm{m} 3$ \\
\hline Esfuerzo a la fluencia & 250 & $\mathrm{MPa}$ \\
\hline Esfuerzo a la tensión & $400-500$ & $\mathrm{MPa}$ \\
\hline Elongación mínima en 50mm & $23 \%$ & \\
\hline Módulo de elasticidad & 200 & $\mathrm{GPa}$ \\
\hline Espesor del material & 0.06 & $\mathrm{~m}$ \\
\hline
\end{tabular}

Tabla 4 Propiedades del acero estructural Fuente: (SUMITEC, 2017)

La geometría del cubo se importó de SolidWorks en formato IGS a la interfaz de ANSYS. Lo primero que se realizó fue definir las condiciones iniciales, como fue los soportes fijos, las fuerzas y momentos que actúan en la estructura del cubo, cabe aclarar que los ejes del software son diferentes a los de referencia de la norma por lo cual se ajustaron para que correspondieran, en este caso se hizo el cambio de ejes como se muestra en la figura 5.

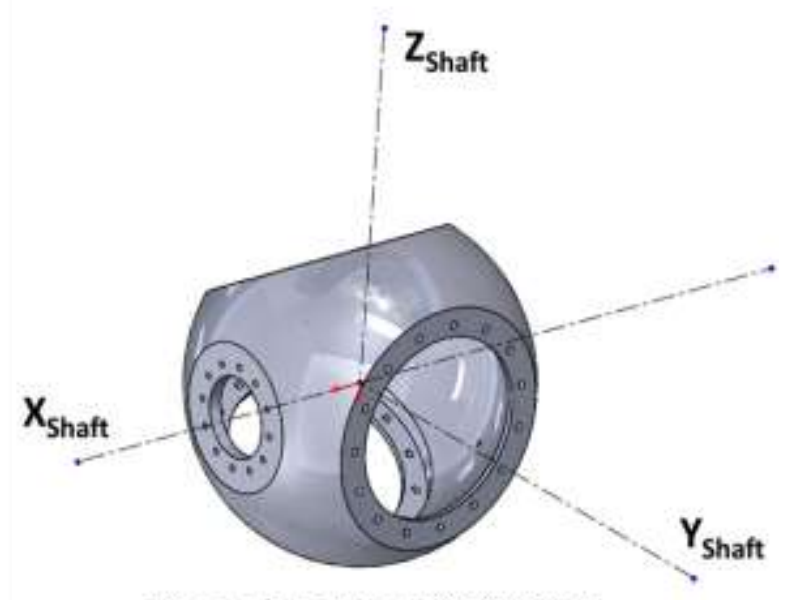

Ejes en la norma IEC 61400-2

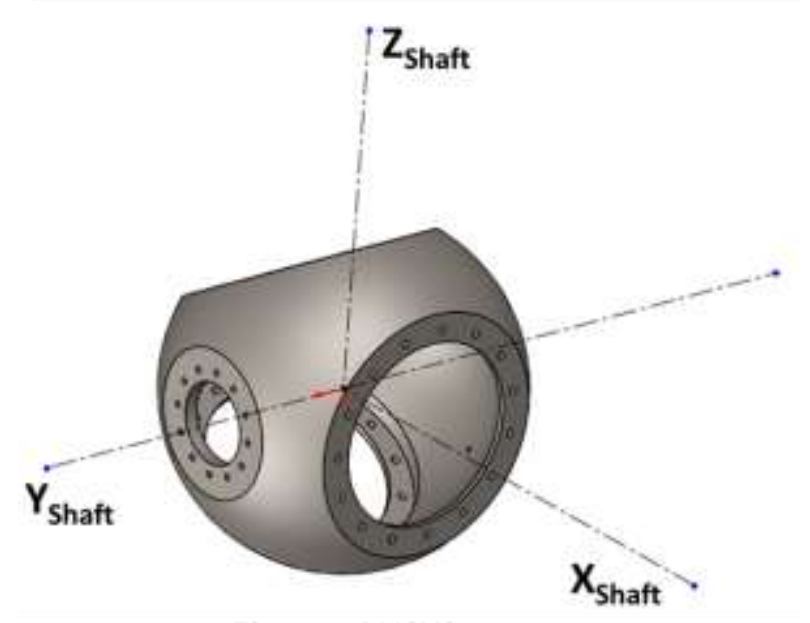

Ejes en ANSYS

Figura 5 Conversión de ejes de ANSYS y de la norma IEC 61400-2

Fuente: Elaboración Propia

Para el caso de carga A, correspondiente a la ecuación 1 y los casos de carga $\mathrm{D}$ y $\mathrm{H}$ de las ecuaciones 7 y 9 respectivamente, para esto las condiciones que se aplicaron, fue colocar un soporte fijo en la cara de conexión con el eje del tren de potencia (color azul, figura 6) y también se colocó una fuerza sobre el eje Y en dirección hacia la torre en las caras donde van las bridas del aspa (color rojo) tal y como se muestra en la figura 6 . 


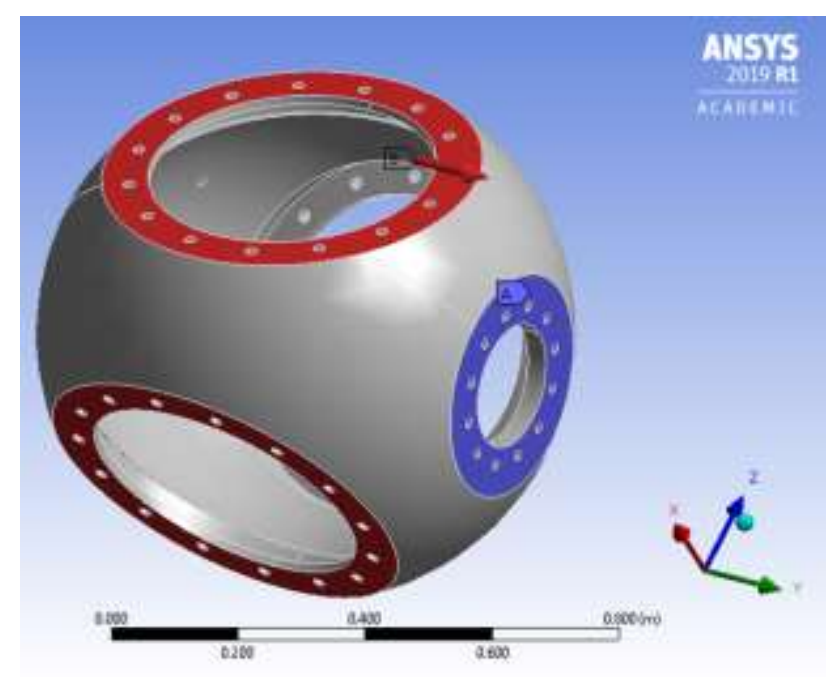

Figura 6 Configuración del caso de carga A, D y H. (Fuerzas sobre el eje X)

Fuente: (ANSYS Workbench R1 ACADEMIC, 2019)

Para el caso de carga A, correspondiente a la ecuación 2, las condiciones iniciales cambiaron colocando los soportes fijos en las caras donde van las bridas de las aspas (color azul, figura 7), mientras el momento se colocó sobre la cara de conexión del eje del tren de potencia (color rojo) tal y como se muestra en la figura 7.

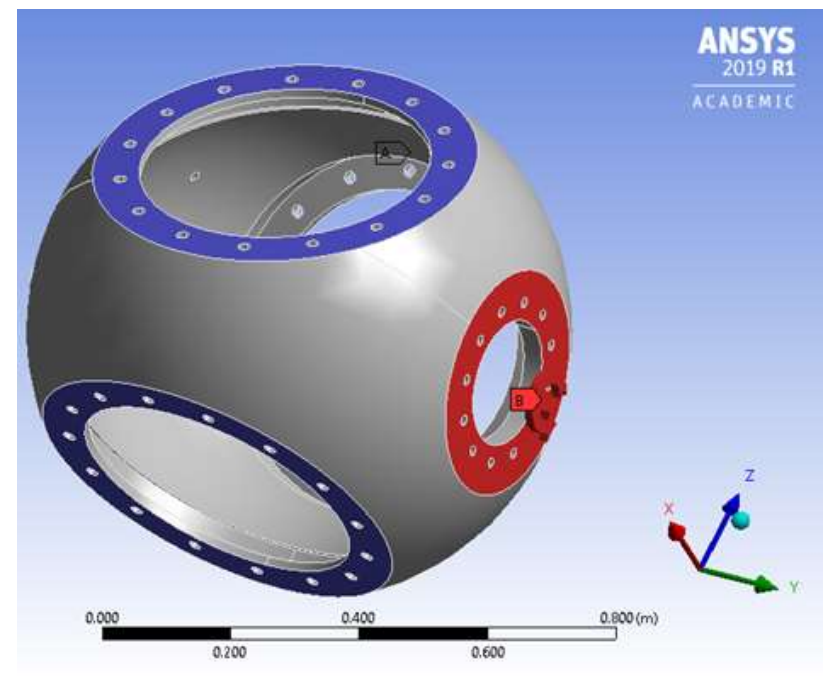

Figura 7 Configuración del caso de carga A (momento en el eje X)

Fuente: (ANSYS Workbench R1 ACADEMIC, 2019)

\section{Resultados}

En esta sección se comenzó con la generación del mallado del cubo para generar el modelo de elementos finitos, el proceso de mallado se comenzó con un tamaño del elemento de $0.1 \mathrm{~m}$ y se aplicó un mallado general a toda la pieza, figura 8 (a). posteriormente se hizo una simulación para verificar la zona de interés de estudio, como se muestra en la figura 8 (b), los elementos que se utilizaron fueron tetraédricos.

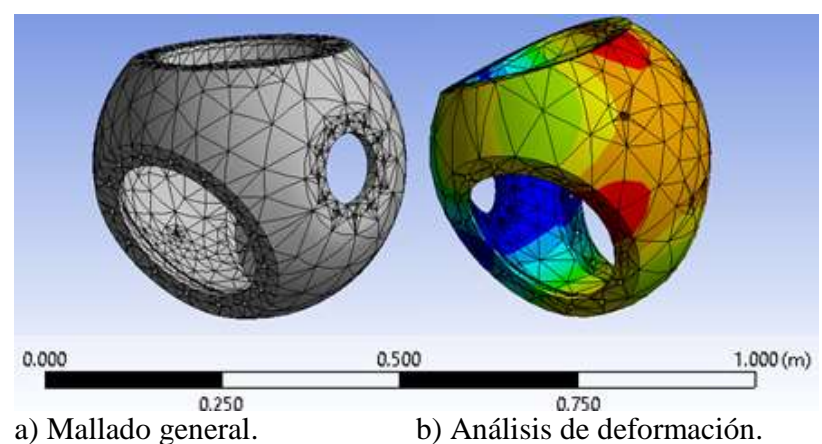

Figura 8 Mallado general del cubo, caso de carga $\mathrm{H}$ Fuente: (ANSYS Workbench RI ACADEMIC, 2019)

Posteriormente, se utilizó la herramienta de mallado de body sizing en donde se configuró el tipo de la esfera de influencia con un radio de $0.5 \mathrm{~m}$ teniendo su origen exactamente en el centro de la conexión del cubo con la flecha del tren de potencia, esto es para el caso de carga A de la ecuación 2, el mallado en esta zona de incidencia se muestra en la figura 9.

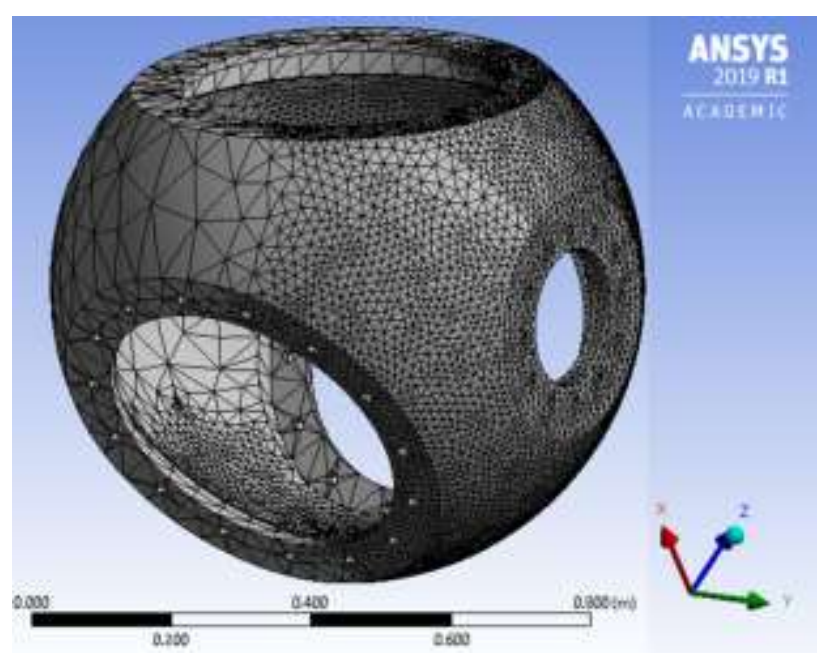

Figura 9 Mallado para el caso de carga A (Momento en $\mathrm{X})$

Fuente: (ANSYS Workbench R1 ACADEMIC, 2019)

Para los casos de carga A, ecuación 1, D y $\mathrm{H}$, la zona de influencia a estudiar es la que se encuentra en la parte posterior del cubo por lo cual se generó la misma configuración de malla del caso pasado. La ubicación de la esfera de influencia se muestra en la figura 10.

COLÍN-MARTINEZ, José Luis, LÓPEZ-GARZA, Víctor, HERNÁNDEZ-ARRIAGA, Isaac y NAVARRO-ROJERO, María Guadalupe. Diseño y simulación preliminar del cubo del rotor para una turbina eólica de 50-kW clase II, de acuerdo a la norma IEC-61400-2. Revista del Diseño Innovativo. 2020 


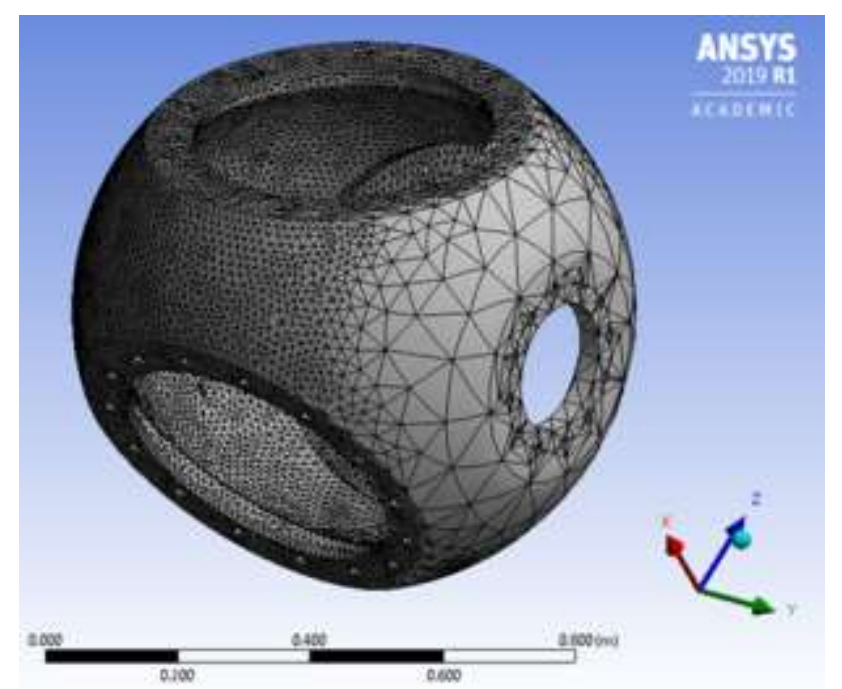

Figura 10 Mallado para los casos de carga A, D y H (Fuerzas en X)

Fuente: (ANSYS Workbench RI ACADEMIC, 2019)

Una vez generada la malla, se fue observando la calidad de los elementos internos de la zona de influencia para verificar las formas tetraédricas de cada elemento en el cual cada vez se cambiaba el tamaño del elemento se hacían más uniformes, como se muestra en la figura 11.

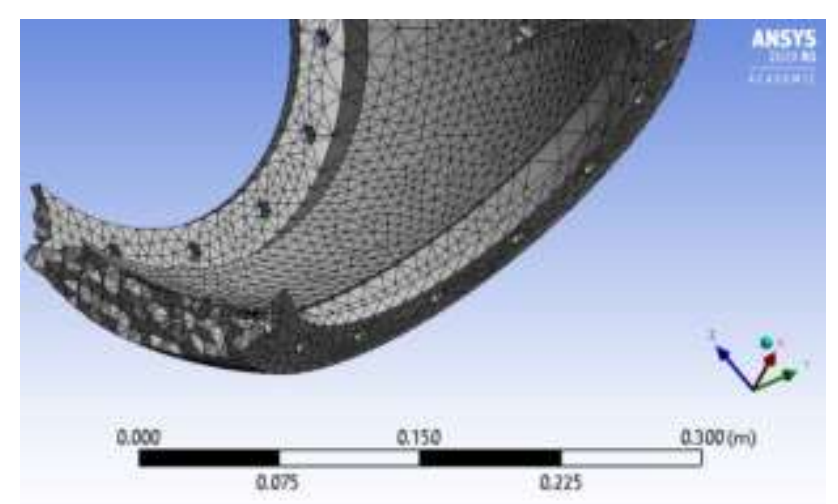

Figura 11 Detalle de la malla interna refinada para el caso de carga A (Momento en Y).

Fuente: (ANSYS Workbench RI ACADEMIC, 2019)

Se realizó el proceso de convergencia de malla, esto para asegurar la solución del problema, se realizaron 10 simulaciones con 3 análisis: el máximo esfuerzo, el esfuerzo equivalente de Von Mises y la deformación, en la tabla 5, se muestran los resultados de las distintas iteraciones. Además, en el gráfico 1 se muestra los resultados de la teoría de falla de Von Mises en el cual se puede observar la convergencia de la malla del caso de carga A, enseguida también se muestran los resultados y graficas de cada uno de los casos de carga.

\begin{tabular}{|r|r|r|r|}
\hline \multicolumn{5}{|c|}{ Caso de carga A: Empuje en el eje X } \\
\hline Elementos & $\begin{array}{l}\text { Máximo } \\
\text { esfuerzo } \\
(\mathrm{Pa})\end{array}$ & $\begin{array}{l}\text { Von } \\
\text { Mises } \\
(\mathrm{Pa})\end{array}$ & $\begin{array}{l}\text { Deformación } \\
(\mathrm{m})\end{array}$ \\
\hline 5268 & 32673 & 135350 & $6.42 \mathrm{E}-08$ \\
\hline 5679 & 33056 & 135350 & $6.50 \mathrm{E}-08$ \\
\hline 5919 & 30796 & 27825 & $6.52 \mathrm{E}-08$ \\
\hline 6353 & 30958 & 27937 & $6.53 \mathrm{E}-08$ \\
\hline 9366 & 33496 & 30933 & $6.65 \mathrm{E}-08$ \\
\hline 11833 & 31907 & 27082 & $6.69 \mathrm{E}-08$ \\
\hline 19390 & 31384 & 29119 & $6.72 \mathrm{E}-08$ \\
\hline 50750 & 31067 & 27082 & $6.76 \mathrm{E}-08$ \\
\hline 51113 & 32207 & 28722 & $6.22 \mathrm{E}-08$ \\
\hline 107001 & 33004 & 29544 & $6.76 \mathrm{E}-08$ \\
\hline
\end{tabular}

Tabla 5 Resultados de las simulaciones del caso de carga A

Fuente: Elaboración Propia

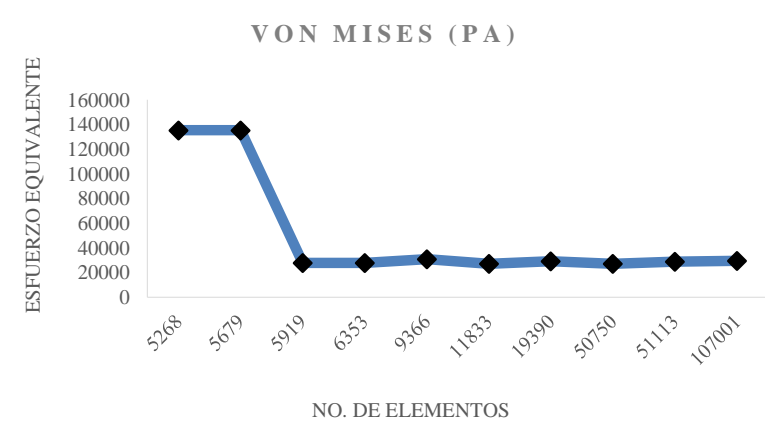

Gráfico 1 Convergencia de malla del caso A: Empuje en el eje $\mathrm{X}$

Fuente: Elaboración propia

En la tabla 6 y en el grafico 2 se muestran los resultados del caso de carga A de la teoría de falla de Von Mises.

\begin{tabular}{|r|r|r|r|}
\hline \multicolumn{5}{|c|}{ Caso de carga A: Momento en el eje X } \\
\hline Elementos & $\begin{array}{l}\text { Máximo } \\
\text { esfuerzo } \\
(\mathrm{Pa})\end{array}$ & $\begin{array}{l}\text { Von } \\
\text { Mises } \\
(\mathrm{Pa})\end{array}$ & $\begin{array}{l}\text { Deformación } \\
(\mathrm{m})\end{array}$ \\
\hline 5878 & 85372 & 30152 & $7.31 \mathrm{E}-08$ \\
\hline 5893 & 85372 & 140620 & $7.29 \mathrm{E}-08$ \\
\hline 6114 & 86097 & 13239 & $7.30 \mathrm{E}-08$ \\
\hline 6491 & 89125 & 141410 & $7.33 \mathrm{E}-08$ \\
\hline 8274 & 105150 & 143680 & $7.34 \mathrm{E}-08$ \\
\hline 10192 & 97111 & 144360 & $7.35 \mathrm{E}-08$ \\
\hline 15153 & 109350 & 153390 & $7.36 \mathrm{E}-08$ \\
\hline 36614 & 127450 & 159820 & $7.35 \mathrm{E}-08$ \\
\hline 72337 & 128850 & 162852 & $7.34 \mathrm{E}-08$ \\
\hline 113658 & 128580 & 165200 & $7.39 \mathrm{E}-08$ \\
\hline
\end{tabular}

Tabla 6 Resultados de las simulaciones del caso de carga A

Fuente: Elaboración Propia 


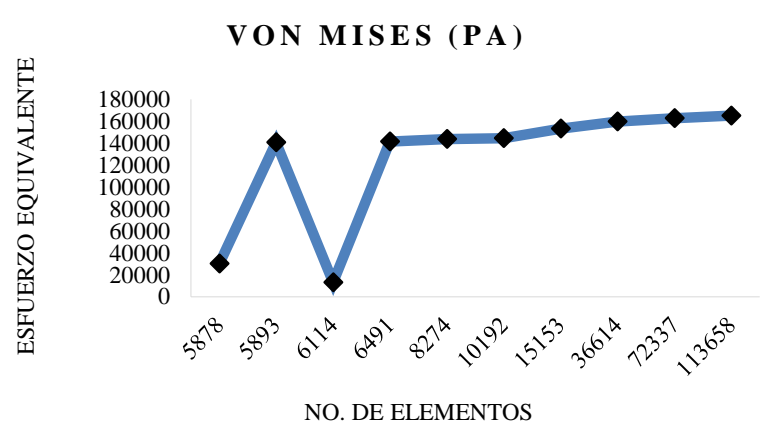

Gráfico 2 Convergencia de malla del caso A: Momento en el eje X.\}

Fuente: Elaboración Propia

En la tabla 7 y en el gráfico 3 se muestran los resultados del caso de carga $\mathrm{D}$ de la teoría de falla de Von Mises.

\begin{tabular}{|r|c|c|c|}
\hline \multicolumn{4}{|c|}{ Caso de carga D: Máximo empuje en el eje X } \\
\hline Elementos & $\begin{array}{c}\text { Máximo } \\
\text { esfuerzo }(\mathrm{Pa})\end{array}$ & $\begin{array}{c}\text { Von Mises } \\
(\mathrm{Pa})\end{array}$ & $\begin{array}{c}\text { Deformación } \\
(\mathrm{m})\end{array}$ \\
\hline 5268 & 4705500 & 4342500 & $9.25 \mathrm{E}-06$ \\
\hline 5679 & 4760700 & 4394100 & $9.37 \mathrm{E}-06$ \\
\hline 5919 & 4435200 & 4007300 & $9.39 \mathrm{E}-06$ \\
\hline 6353 & 4458500 & 4023500 & $9.41 \mathrm{E}-06$ \\
\hline 9366 & 4825100 & 4455000 & $9.58 \mathrm{E}-06$ \\
\hline 11833 & 4595200 & 4165100 & $9.63 \mathrm{E}-06$ \\
\hline 19390 & 4133700 & 4715600 & $9.67 \mathrm{E}-06$ \\
\hline 50750 & 4474200 & 4900400 & $9.73 \mathrm{E}-06$ \\
\hline 51113 & 4538400 & 4880500 & $8.96 \mathrm{E}-06$ \\
\hline 107001 & 4876300 & 4792800 & $9.73 \mathrm{E}-06$ \\
\hline
\end{tabular}

Tabla 7 Resultados de las simulaciones del caso de carga D

Fuente: Elaboración Propia

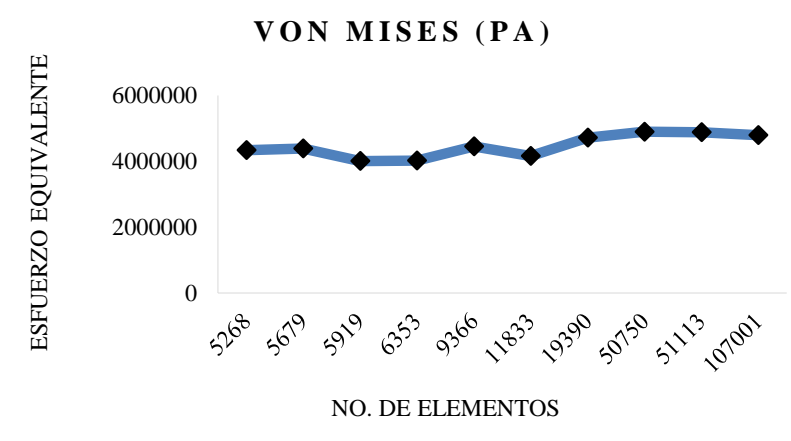

Gráfico 3 Convergencia de malla del caso D: Máximo empuje en el eje $\mathrm{X}$

Fuente: Elaboración Propia

En la tabla 8 y en el gráfico 4 se muestran los resultados del caso de carga $\mathrm{H}$ de la teoría de falla de Von Mises.

\begin{tabular}{|r|c|c|c|}
\hline \multicolumn{4}{|c|}{ Caso de carga H: Máximo empuje en el eje X } \\
\hline Elementos & $\begin{array}{c}\text { Máximo } \\
\text { esfuerzo } \\
(\mathbf{P a})\end{array}$ & $\begin{array}{c}\text { Von } \\
\text { Mises } \\
(\mathbf{P a})\end{array}$ & $\begin{array}{c}\text { Deformació } \\
\text { n (m) }\end{array}$ \\
\hline 5268 & 26029000 & 24021000 & $5.12 \mathrm{E}-05$ \\
\hline 5679 & 26334000 & 24306000 & $5.18 \mathrm{E}-05$ \\
\hline 5919 & 24534000 & 22166000 & $5.20 \mathrm{E}-05$ \\
\hline 6353 & 24663000 & 22256000 & $5.20 \mathrm{E}-05$ \\
\hline 9366 & 26685000 & 24643000 & $5.30 \mathrm{E}-05$ \\
\hline 11833 & 25418000 & 23045000 & $5.33 \mathrm{E}-05$ \\
\hline 19390 & 28397000 & 26086000 & $5.35 \mathrm{E}-05$ \\
\hline 50750 & 24749000 & 21575000 & $5.38 \mathrm{E}-05$ \\
\hline 51113 & 25657000 & 21578000 & $4.95 \mathrm{E}-05$ \\
\hline 107001 & 26148000 & 21146000 & $5.38 \mathrm{E}-05$ \\
\hline
\end{tabular}

Tabla 8 Resultados de las simulaciones del caso de carga $\mathrm{H}$

Fuente: Elaboración Propia

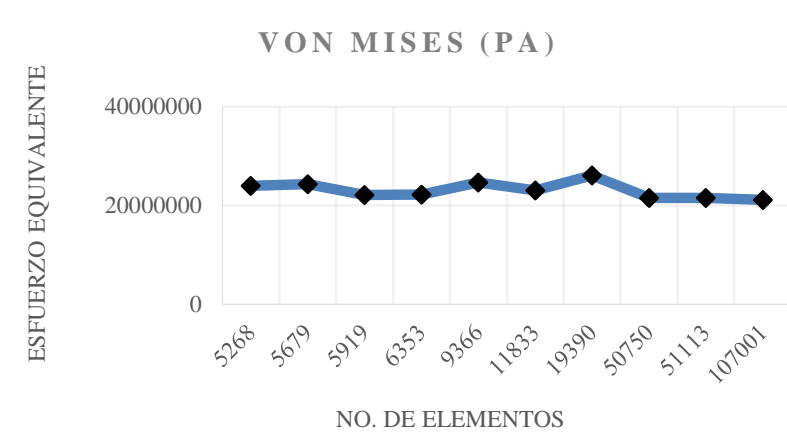

Gráfico 4 Convergencia de malla del caso H: Máximo empuje en el eje $\mathrm{X}$

Fuente: Elaboración Propia

Una vez que se obtiene la convergencia de la malla, se pueden asegurar que los resultados de cada caso de carga son confiables y se pueden tomar estos datos para el rediseño posterior del cubo. A continuación, se muestra una simulación de ejemplo del caso de carga $\mathrm{H}$, la cual es la hipótesis con el valor más alto de fuerza que se da en el eje $\mathrm{X}$, los desplazamientos máximos de este caso se muestran en la figura 12.

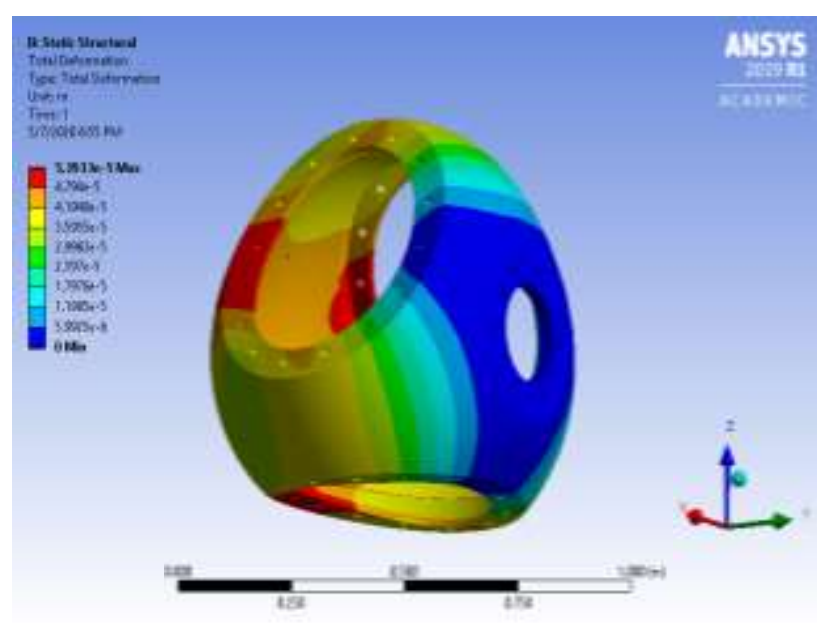

Figura 12 Resultado del análisis de deformación del caso de carga D

Fuente: (ANSYS Workbench R1 ACADEMIC, 2019) 
La figura 13 muestra la simulación del esfuerzo máximo principal del caso de carga $\mathrm{D}$, en donde se muestran los resultados.

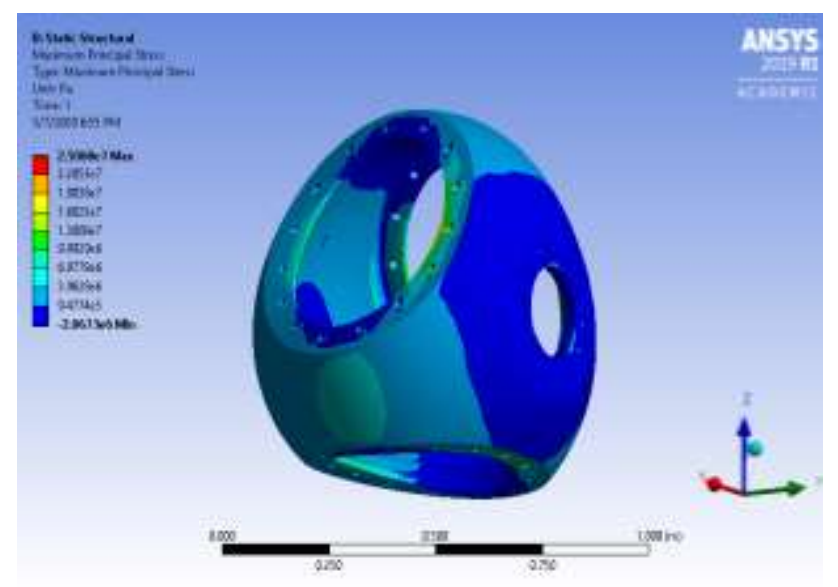

Figura 13 Resultado del esfuerzo máximo del caso de carga D

Fuente: (ANSYS Workbench R1 ACADEMIC, 2019)

La figura 14 muestra los esfuerzos de la teoría de falla de Von Mises del caso de carga D.

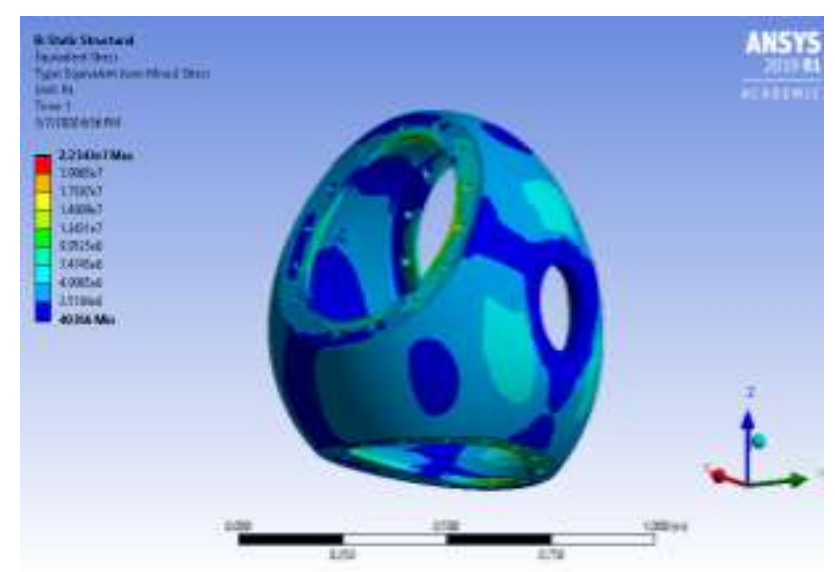

Figura 14 Resultado del análisis de la teoría de falla de Von Mises del caso de carga D

Fuente: (ANSYS Workbench RI ACADEMIC, 2019)

\section{Agradecimiento}

A la gerencia de Turbomaquinaria y al posgrado de CIATEQ A.C. por las facilidades otorgadas para el desarrollo del presente trabajo. Así como al CONACYT por las becas otorgadas a los alumnos de maestría.

\section{Conclusiones}

Los resultados obtenidos son satisfactorios para el material que se eligió para hacer la simulación, sin embargo, se deben de hacer más simulaciones del cubo considerando otro tipo de acero y una vez que se tenga el diseño de los componentes del aerogenerador (Aspas, tren de potencia góndola, sistemas de control etc.) se podrá dimensionar el cubo tomando en cuenta el diseño para el ensamble con otros componentes auxiliares, para que después se puedan afinar detalles de diseño.

Las simulaciones se realizaron con una licencia académica por lo que tenía un límite de elementos a analizar, por lo cual se puede utilizar una licencia comercial con la que cuenta CIATEQ, esto ayudaría a generar una malla más detallada una vez que se tenga el diseño final del componente.

Por los resultados obtenidos podemos concluir que la pieza es demasiado robusta ya que los desplazamientos que existen son demasiado pequeños por lo que se debe de hacer una optimización de la simulación para llegar al dimensionamiento mínimo cumpliendo los factores de seguridad establecidos por la norma.

\section{Referencias}

Aguilar-Acevedo, F. M.-V.-L.-P.-S.-B. (2020). Modelado Euler-Lagrange del rotor de un.

AMDEE. (2018). Asociación Mexicana de Energía Eólica . Obtenido de https://amdee.org/ Anil Mital, S. N. (Noviembre de 2017). Ingeniería de Manufactura en el siglo XXI.

ANSYS Workbench R1 ACADEMIC. (2019).

Buenestado, J. F. (2017). ANÁLISIS Y DISEÑO DE UN AEROGENERADOR MEDIANTE SIMULACIÓN 3D DE DINÁMICA COMPUTACIONAL DE FLUIDOS. Madrid España.

Claudia Marcela Sánchez R, C. J. (14 de Octubre de 2005). Conceptos de diseño para manufactura (DFM) de piezas microfundidas.

DE OLIVEIRA, V. H. (2020). DIMENSIONAMENTO GEOTÉCNICO DA FUNDAÇÃO DE UMA TURBINA EÓLICA. 
DNV RISO. (2002). Guidelines for desing of wind turbines. Denmark.

Fernández Guevara, A. (2020). Diseño de un parque híbrido de $15 \mathrm{MW}$ aplicado en la provincia de Celendín, región Cajamarca.

Funes Castro, S. (2020). Caracterización de una turbina mareomotriz de eje vertical.

IEC. (2013). INTERNATIONAL STANDRD IEC 61400. Suiza.

IFM. (2017). Sistema pitch eléctrico en un aerogenerador sin multiplicadora. Recuperado el Marzo de 2020, de http://eval.ifmelectronic.com/ifmmx/web/apps-byindustry/cat_060_010_010.html

\section{J.F. Manwell, J. M. (2009). WIND ENERGY} EXPLANINED. UK: WILEY.

Krishnappa, L. (2015). Fatigue Analysis of a 7.5 MW wind turbine rotor hub. Hannover, Alemania.

Scitechdaily. (2 de January de 2020). Obtenido de Successful Test for Superconducting 3.6Megawatt Wind Turbine: https://scitechdaily.com/successful-test-forsuperconducting-3-6mw-wind-turbine/

SolidWorks. (2016).

SUMITEC. (2017). Acero estructural A36. México.

Warmerdam, M. P. (2017-2018). Aerodinámica de aerogenerador de eje horizontal de $500 \mathrm{~kW}$. La Rioja España.
COLÍN-MARTINEZ, José Luis, LÓPEZ-GARZA, Víctor, HERNÁNDEZ-ARRIAGA, Isaac y NAVARRO-ROJERO, María Guadalupe. Diseño y simulación preliminar del cubo del rotor para una turbina eólica de 50-kW clase II, de acuerdo a la norma IEC-61400-2. Revista del Diseño Innovativo. 2020 